\title{
Mars Climate Engineering using Orbiting Solar Reflectors
}

\author{
C.R. McInnes
}

Advanced Space Concepts Laboratory, Department of Mechanical Engineering, University of Strathclyde, Glasgow, G1 1XJ, Scotland, UK

\section{Introduction}

Terrestrial geo-engineering is currently being explored as a largescale venture to mitigate against rapid terrestrial climate change due to anthropogenic carbon emissions. A range of schemes have been proposed, including the use of orbiting solar reflectors to reduce solar insolation to compensate for increased radiative forcing of the climate (Early 1989; Angel 2006). While the scale of endeavour required to deploy geo-engineering schemes is impressive, on an even more ambitious scale the same technologies which can be envisaged to engineer the Earth's climate can be scaled to engineer the climate of Mars. Such terraforming schemes (engineering an Earth-like cli- 
mate) have long been discussed, although the concept became somewhat more mainstream with the work of Sagan and others (Sagan 1961, 1973). Bioengineering schemes have been proposed, including the delivery of customized organisms to convert carbon dioxide to oxygen in the atmosphere of Mars, and darkening the Martian polar caps to reduce their albedo, again using customized organisms. Halocarbons synthesised on Mars have also been considered as a tool to quickly raise the surface temperature and so liberate trapped carbon dioxide (Gerstell et al. 2001; Badescu 2005).

More direct terraforming schemes have been proposed for Mars, including the use of orbiting solar reflectors to increase total solar insolation. Such reflectors can be used to increase the mean surface temperature through direct radiative forcing, or can attempt to catalyze favourable climatic transitions by preferentially heating the polar caps in an attempt to release frozen carbon dioxide. Zubrin and McKay estimate that a rise in polar temperature of order $4 \mathrm{~K}$ may be sufficient to drive a transition to a new climate state in which the polar caps have evaporated, delivering an atmosphere with a surface pressure of 50-100 mb, as the first step of a longer term terraforming process. It is estimated that sufficient carbon dioxide exists in both the polar caps and regolith to ultimately raise the surface pressure to $300-600 \mathrm{mb}$. The solar reflectors to produce such a transition are envisaged to be in static equilibria high above the poles of the planet (Zubrin and McKay 1997), or in displaced polar orbits (McInnes 2002). The reflector system proposed by Zubrin was to be used solely to heat the polar caps, while the much larger systems 
discussed by both Birch (1992) and Fogg (1992) were seen as providing an increase in total insolation, averaged over the planetary surface. These reflectors are envisaged as part of a larger terraforming effort, with the reflectors used to increase the total planetary insolation by as much as $30 \%$.

As has been noted elsewhere (Maunter and Parks 1990), the main advantage of using large solar reflectors for planetary engineering (geo-engineering or terraforming) is the vast energy leverage obtained in a relatively short time. The total energy intercepted by the reflector quickly grows beyond the energy required for its fabrication, leading to a highly efficient tool for climate modification. The use of reflectors with a deployed area of order $10^{12} \mathrm{~m}^{2}$ and a mass of order $10^{10} \mathrm{~kg}$ at the Earth-Sun $\mathrm{L}_{1}$ point has been explored (McInnes 2007) as a means of mitigating against anthropogenic climate change (Fig 6.1). As will be seen, the required reflector area for Mars terraforming is of order $10^{13} \mathrm{~m}^{2}$ (and mass of order $10^{10}$ $\mathrm{kg})$. A single large reflector is clearly impractical so that a large number of smaller reflectors are envisaged, fabricated from asteroid resources and distributed azimuthally in a uniform ring around a displaced polar orbit to provide the same total reflective area.

Families of displaced polar orbits suitable for Mars terraforming have been investigated in some detail for solar sails and a range of relatively near-term mission applications proposed (McInnes 1991; McInnes and Simmons 1992; McInnes 1999). Such displaced orbits can also be exploited for large solar reflectors, where the reflector orientation is defined a priori to ensure back reflection 
of solar radiation onto Mars, as discussed by Fogg (1995). The principal advantage of these orbits is that the reflector is never in eclipse, and it can be shown that the effective transfer of energy per orbit is greater than for circular orbits in the ecliptic plane of Mars. In addition, the strong solar radiation pressure perturbation on the reflector is managed by the out-of-plane component of gravitational force. In this chapter the orbital dynamics of displaced solar reflectors is investigated in detail, with a complete family of orbits suitable for Mars terraforming identified. The linear stability characteristics of this family of orbits are also investigated, and a stable sub-group of orbits identified with the resulting orbits locked in a Sunsynchronous state. This family of passively stable, Sun-synchronous orbits appears to offer significant benefits for large Mars solar reflectors.

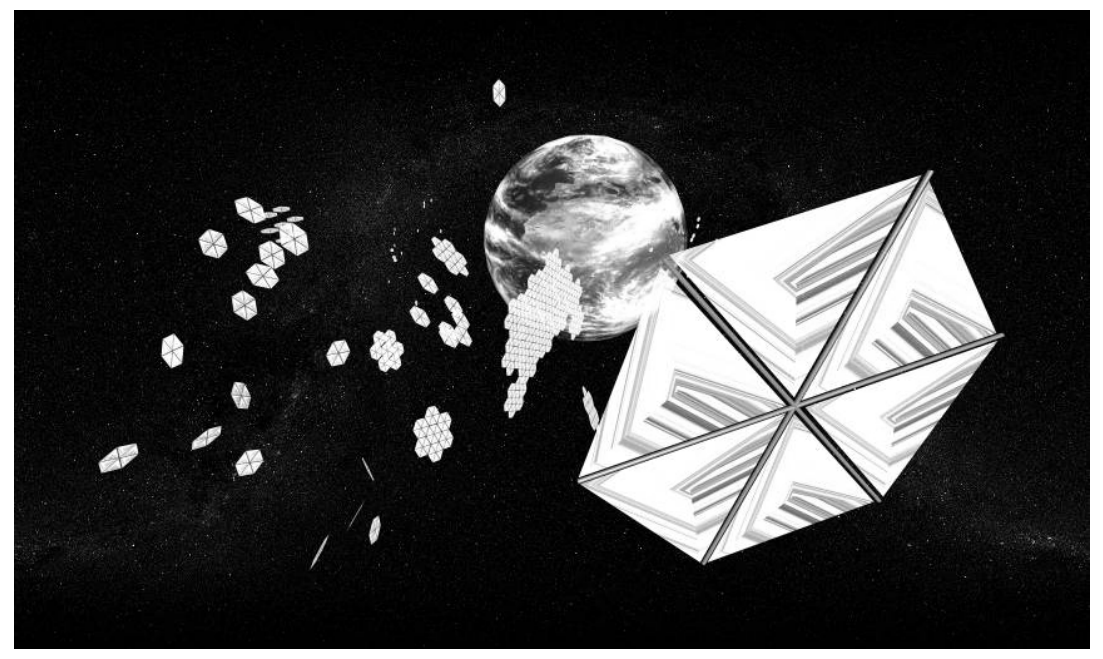

Fig. 6.1. Swarm of reflectors for terrestrial geo-engineering (Dario Izzo ESA). 
In addition to displaced orbits, the use of static equilibria for Mars solar reflectors proposed by Zubrin is also discussed (Zubrin and McKay 1997). At these locations on the night-side of Mars, the solar radiation pressure force exerted on the solar reflector exactly balances the local gravitational force. The efficiency of these locations for increasing planetary insolation is discussed. It has been shown that even for an ideal reflector, the reflector orientation which is required to ensure static equilibrium leads to only half of the radiation reflected being intercepted by the planetary body, while the remainder is lost. For a more realistic reflector with non-perfect optical reflectivity it is found that the problem is significantly worse (McInnes, 2000). In fact, the orientation required for a non-ideal reflector to be maintained in static equilibrium can lead to all of the reflected radiation being directed away from the planetary body. Therefore, there are fundamental difficulties associated with the use of static equilibria for Mars terraforming.

\section{Mars Climate Engineering}

As discussed earlier, solar reflectors can be envisaged as planetary engineering tools which can be used to drive the mean surface temperature of Mars upwards by direct radiative forcing, or by attempting to liberate frozen carbon dioxide from the polar caps (Oberg 1981; McKay et al. 1991; Birch 1992; Fogg 1995; Zubrin and McKay 1997). Here, it will be assumed that solar reflectors are used to increase the planetary insolation by back reflecting solar radia- 
tion, averaged across the entire planetary surface. The approximate requirements for a solar reflector to increase the total insolation of Mars can therefore be determined using a simple thermal balance model for the mean planetary surface temperature. For an incident solar flux $S$, the planetary black-body temperature $T_{B}$ can be obtained from a heat balance such that $(1-\alpha) \pi R^{2} S=4 \pi R^{2} \sigma T_{B}{ }^{4}$ so that the mean surface temperature $T_{M}$ may be written as

$$
T_{M}=\Delta T_{G}+\left[\frac{(1-\alpha)}{4 \sigma} S\right]^{1 / 4}
$$

where $\alpha$ is the Bond albedo of Mars, $R$ is the planetary radius (3390 $\mathrm{km})$ and $\sigma$ is the Stefan-Boltzmann constant $\left(5.67 \times 10^{-8} \mathrm{Wm}^{-2} \mathrm{~K}^{-4}\right)$. The term $\Delta T_{G}$ accounts for any modest increase in the mean surface temperature over the black-body temperature due to current atmospheric greenhouse warming. For an incident solar flux of $589 \mathrm{Wm}^{-2}$ and an albedo of 0.25 , the black-body temperature is found to be 210 $\mathrm{K}$. This is only slightly cooler than the mean surface temperature of $217 \mathrm{~K}$, the discrepancy being due to atmospheric carbon dioxide.

In order to directly increase the mean surface temperature to that required for terraforming by radiative forcing would require a significant increase in planetary insolation. However, feedback processes are likely to be of benefit and may allow a more modest increase in solar insolation to achieve a large rise in mean surface temperature. For example, as the mean surface temperature rises due to solar heating, carbon dioxide from the polar caps may be liberated, leading to a positive feedback as the Martian atmosphere is 
thickened with greenhouse gases. In a synergetic scenario for terraforming Mars an increase in solar insolation of order 30\% is envisaged (Fogg 1992), which is coupled with other activities to drive the mean surface temperature upwards.

The total planetary insolation of Mars is simply $\pi R^{2} S$ which corresponds to a thermal input of $2.13 \times 10^{16} \mathrm{~W}$. To increase the planetary insolation by some fraction $\lambda$, the heat balance is modified such that $(1-\alpha) \pi R^{2} S+\lambda \pi R^{2} S=4 \pi R^{2} \sigma T_{B}{ }^{4}$. The new mean surface temperature can therefore be written as

$$
T_{M}=\Delta T_{G}+\left[\frac{(1-\alpha+\lambda)}{4 \sigma} S\right]^{1 / 4}
$$

An enhancement of total insolation by $30 \%$ requires an additional $6.38 \times 10^{15} \mathrm{~W}$ to be delivered by the solar reflectors. With this additional thermal input Eq. (6.2) shows that the black-body temperature of the planet rises to $228 \mathrm{~K}$, providing only a modest direct increase in mean surface temperature, although again climatic feedback is anticipated to drive the mean surface temperature upwards (Fogg 1992).

\section{Solar Reflector Orbit Selection}

\subsection{Circular Orbit}

In order to assess candidate orbits for solar reflectors, an evaluation of three possibilities will be conducted: a circular orbit in the ecliptic 
plane of Mars, a polar orbit normal to the ecliptic plane of Mars and static equilibria high above the poles of Mars. For a reflector on a circular orbit, with the reflector position defined by polar angle $\theta$ relative to the Sun-line, the reflector is oriented such that $\alpha=\theta / 2$ in order that reflected solar radiation is directed towards the surface of Mars, as shown in Fig. 6.2. For a reflector of area $A$, on a circular orbit of period $T$, the mean energy transfer $\Delta E$ per unit time is therefore given by

$$
\left\langle\frac{\Delta E}{T}\right\rangle_{C}=\frac{1}{T} \int_{0}^{T} S A \cos (\theta / 2) d t
$$

However, the orbital angular velocity $\omega=d \theta / d t$ is related to the orbit period $T$ such that $\omega=2 \pi / T$ so that

$$
\left\langle\frac{\Delta E}{T}\right\rangle_{C}=\frac{S A}{\omega T} \int_{-\pi}^{+\pi} \cos (\theta / 2) d \theta
$$

Therefore, performing the integration, the mean energy transfer $\Delta E$ per unit time is given by

$$
\left\langle\frac{\Delta E}{T}\right\rangle_{C}=\frac{2}{\pi} S A
$$

It can be seen from Eq. (6.5) that approximately $64 \%$ of the maximum available solar energy $S A$ is transferred to the surface of Mars by a reflector on a circular orbit. 


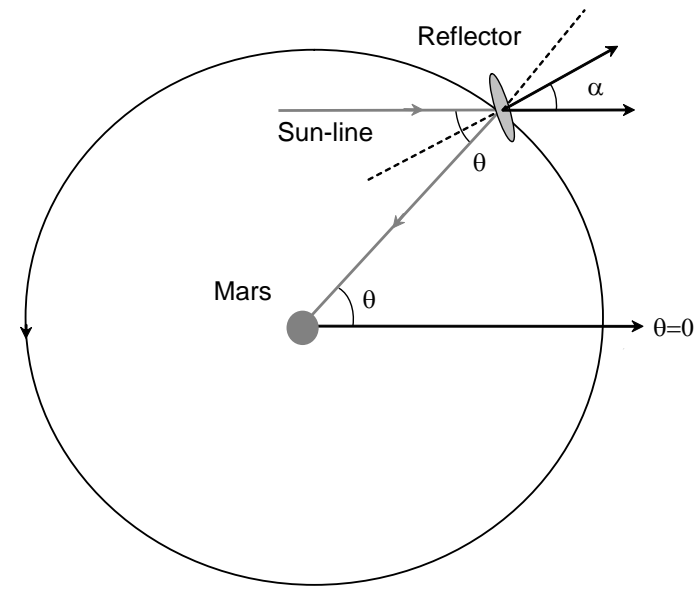

Fig. 6.2. Solar reflector on a circular orbit in the ecliptic plane of Mars

\subsection{Polar Orbit}

For comparison, a circular polar orbit normal to the ecliptic plane of Mars will now be considered, as shown in Fig. 6.3. Then, the reflector orientation can be fixed as the reflector orbits normal to the Sunline. This fixed orientation provides an additional benefit over orbits in the ecliptic plane. The mean energy transfer $\Delta E$ per unit time for a polar orbit is therefore given by

$$
\left\langle\frac{\Delta E}{T}\right\rangle_{P}=\frac{S A}{\omega T} \int_{0}^{2 \pi} \cos (\alpha) d \theta
$$

In order that the reflected solar radiation is directed toward the surface of Mars (along the terminator) the reflector attitude $\alpha$ is fixed at $\alpha=\pi / 4$ so that 


$$
\left\langle\frac{\Delta E}{T}\right\rangle_{P}=\frac{1}{\sqrt{2}} S A
$$

It can be seen that approximately $71 \%$ of the maximum available solar energy $S A$ is transferred to the surface pf Mars. Evaluating the effectiveness of the circular and polar orbits it can be seen that

$$
\left\langle\frac{\Delta E}{T}\right\rangle_{C}<\left\langle\frac{\Delta E}{T}\right\rangle_{P}
$$

so that polar orbits are in principle more efficient. A family of displaced polar orbits will be considered later in Section 4. These orbits are able to manage momentum transfer to the reflectors from solar radiation pressure, which would otherwise lead to secular perturbations on the reflector orbit.

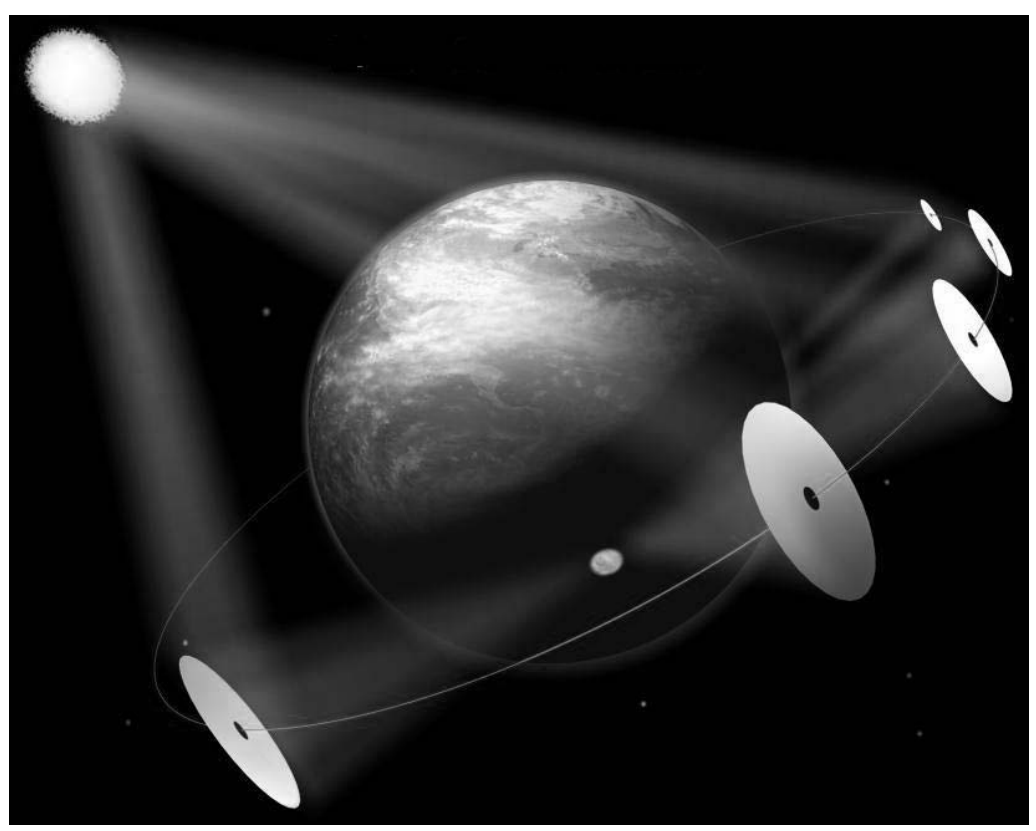

Fig. 6.3. Solar reflectors on polar orbits (Aerospace Systems Ltd). 


\subsection{Static Equilibria}

An alternative to a Keplerian orbit about Mars is non-orbiting static equilibria, as discussed by Zubrin and McKay (1997), based on a concept by Forward (1991). These equilibria require that the solar radiation pressure force exerted on the reflector is directly balanced by the local gravitational force leading to a static equilibrium high above the poles. While a detailed three-body analysis of such equilibria has been performed (McInnes et al. 1994), for static equilibria close to Mars (approximating a 2-body rather than 3-body problem) the reflector normal must be directed along the Mars-reflector line to ensure the correct force balance, assuming an ideal reflector. However, the orientation required for a non-ideal reflector to be maintained in static equilibrium leads to most of the reflected radiation being directed away from the planetary body. Therefore, static equilibria do not appear to be a suitable location for Mars solar reflectors (McInnes, 2002).

\section{Families of Displaced Polar Orbits}

In order to re-direct solar radiation to increase the total planetary insolation, reflectors must be deployed on suitable orbits, as discussed in Section 3. One of the key issues is management of the momentum accumulated by the reflectors due to solar radiation pressure, which poses difficulties for reflectors deployed directly in Keplerian orbit about Mars. In addition, since the reflectors act as mirrors, they will project an image of the solar disk onto the surface of Mars. At the 
distance of Mars from the Sun, the solar disk subtends an angle $\theta$ of order $0.01 \mathrm{rad}$. Therefore, a reflector at distance $d$ from the surface of Mars will project a spot of diameter of order $D \sim d \theta$, assuming a perfectly flat reflecting surface. In order that the spot size is less than the diameter of Mars, the distance $d$ must be less than $1.4 \times 10^{6} \mathrm{~km}$, a distance comparable to that to the $\mathrm{L}_{1}$ point. However, locations near the $\mathrm{L}_{1}$ point are not attractive due to the long path length the reflected radiation must traverse and consequently the extremely high pointing accuracy required for the solar reflectors. In addition, in order to establish an artificial equilibrium point using solar radiation pressure near $L_{1}$, the aspect angle of the reflectors is rather large, leading to a small projected reflector area (McInnes et al. 1994).

Artificial equilibria between Mars and the $\mathrm{L}_{2}$ point have been considered by Zubrin, for heating the poles of Mars (Zubrin and McKay 1997). However, as discussed in Section 3.3, when a reflector with non-ideal reflectivity is considered the required aspect angle of the reflector is such that the re-direct radiation is in fact largely unable to illuminate the poles (McInnes 2002).

As noted earlier, reflectors in orbit about Mars will be strongly perturbed by solar radiation pressure. An attractive family of orbits for solar reflectors are displaced orbits which can be generated by orienting the reflector such that a component of the solar radiation pressure induced force exerted on the reflector is directed normal to the orbit plane (McInnes and Simmons 1992). These orbits are therefore circular and near polar, but are displaced behind 


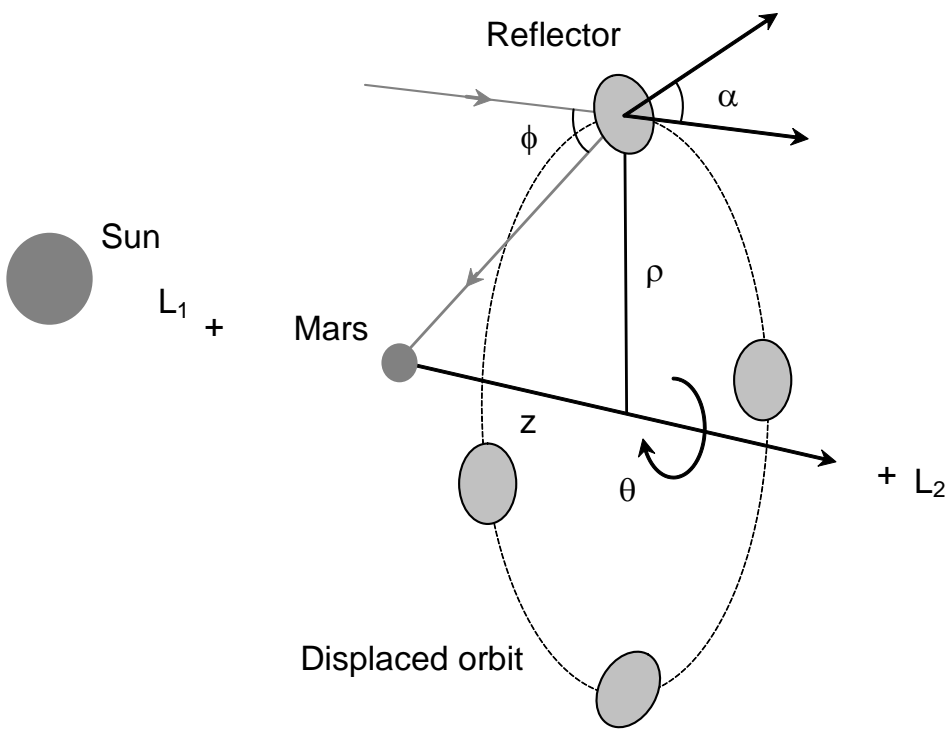

Fig. 6.4. Solar reflectors on a displaced polar orbit along the Sun-line.

Mars in the anti-Sun direction, as shown in Fig. 6.4 for a reflector orbit radius $\rho$ and displacement distance $z$. The momentum accumulated by the reflector due to solar radiation pressure is offset by the z-component of the local gravitational force. Using a two-body analysis, the requirements for such displaced orbits can be investigated. However, it is found that such orbits can also be established using a full three-body analysis of the dynamics of the problem (Bookless and McInnes 2004).

Using cylindrical polar coordinates, the equations of motion for an ideal specular reflector can be written as

$$
\ddot{\rho}-\rho \dot{\theta}^{2}=-\frac{\rho}{r}\left(\frac{\mu}{r^{2}}\right)+a \cos ^{2} \alpha \sin \alpha
$$




$$
\begin{gathered}
\rho \ddot{\theta}+2 \dot{\rho} \dot{\theta}=0 \\
\ddot{z}=-\frac{z}{r}\left(\frac{\mu}{r^{2}}\right)+a \cos ^{3} \alpha
\end{gathered}
$$

where $\mu$ is the product of the gravitational constant and the mass of Mars. The reflector orientation is defined by a pitch angle $\alpha$ relative to the Sun-line and the acceleration $a$ induced by solar radiation pressure is defined by $a=2 P / \sigma$ where $P$ is the solar radiation pressure at the distance of Mars from the Sun $\left(1.964 \times 10^{-6} \mathrm{Nm}^{-2}\right)$ and $\sigma$ is the mass per unit area of the reflector. It is assumed that $a$ is constant (although the magnitude of the solar radiation pressure induced acceleration scales as $\cos ^{2} \alpha$ ). For a circular displaced orbit it is required that $\ddot{\rho}=0$ and $\ddot{z}=0$. Therefore, defining the orbital angular velocity $\omega=\dot{\theta}$ it can be shown that the required reflector pitch angle and acceleration can be written as

$$
\begin{aligned}
& \tan \alpha=\frac{\rho}{z}\left[1-\left[\frac{\omega}{\tilde{\omega}}\right]^{2}\right], \tilde{\omega}^{2}=\frac{\mu}{r^{3}} \\
& a=\tilde{\omega}^{2}\left[1+\left[\frac{\rho}{z}\right]^{2}\left[1-\left[\frac{\omega}{\widetilde{\omega}}\right]^{2}\right]^{2}\right]^{3 / 2} z
\end{aligned}
$$

For the reflector to direct reflected light towards Mars, it is clear from Fig. 6.3 that the reflector pitch angle $\alpha$ is related to $\phi$ such that $\alpha=\phi / 2$. Assuming a perfect specular reflector, an image of the solar disk will then be projected onto Mars, with the image centre on the 
Mars-reflector line. The required reflector pitch angle is therefore defined as

$$
\tan \alpha=\tan \left[\frac{1}{2} \tan ^{-1}\left[\frac{\rho}{z}\right]\right]
$$

Since this orientation is defined a priori, the orbital angular velocity of the reflector must now be a variable of the problem in order to ensure correct illumination of Mars by the reflector. Using Eq. (6.10a) it can be seen that the orbital angular velocity required for this desired reflector orientation is given by

$$
\omega=\widetilde{\omega}\left[1-\left[\frac{z}{\rho}\right] \tan \alpha\right]^{1 / 2}
$$

The corresponding reflector acceleration can now be obtained from Eq. (6.10b). A section of the surfaces of constant reflector acceleration generated by this family of orbits is shown in Fig. 6.5 in the $\rho$-z plane. Each point on a surface corresponds to a displaced orbit with some radius $\rho$ and displacement distance $z$ in the anti-Sun direction. It can be seen that the surfaces of constant reflector acceleration have a rotational symmetry about the Sun-line, and that for a given reflector acceleration $a$ an orbit with a large radius and small displacement is possible $\left(\alpha \sim 45^{\circ}\right)$, or an orbit with a small radius and large displacement $\left(\alpha \sim 0^{\circ}\right)$. Clearly, displaced orbits with a small pitch angle $\alpha$ are desirable to maximise the projected reflector area. However, orbits with large displacements are found to be unstable, as will be seen later in Section 5. An orbit with a relatively modest 
displacement is still able to offset the momentum accumulated by the reflector due to solar radiation pressure by the z-component of the local gravitational force, while the orbit remains Sunsynchronous with the orbit plane always normal to the Sun-Mars line. While not considered further here, it is noted that if the reflector orbit period is chosen to be an integer number of sidereal days, the spot projected onto the surface of Mars by the solar reflector will follow a repeat ground track. This may have some importance if certain regions are to be preferentially heated.

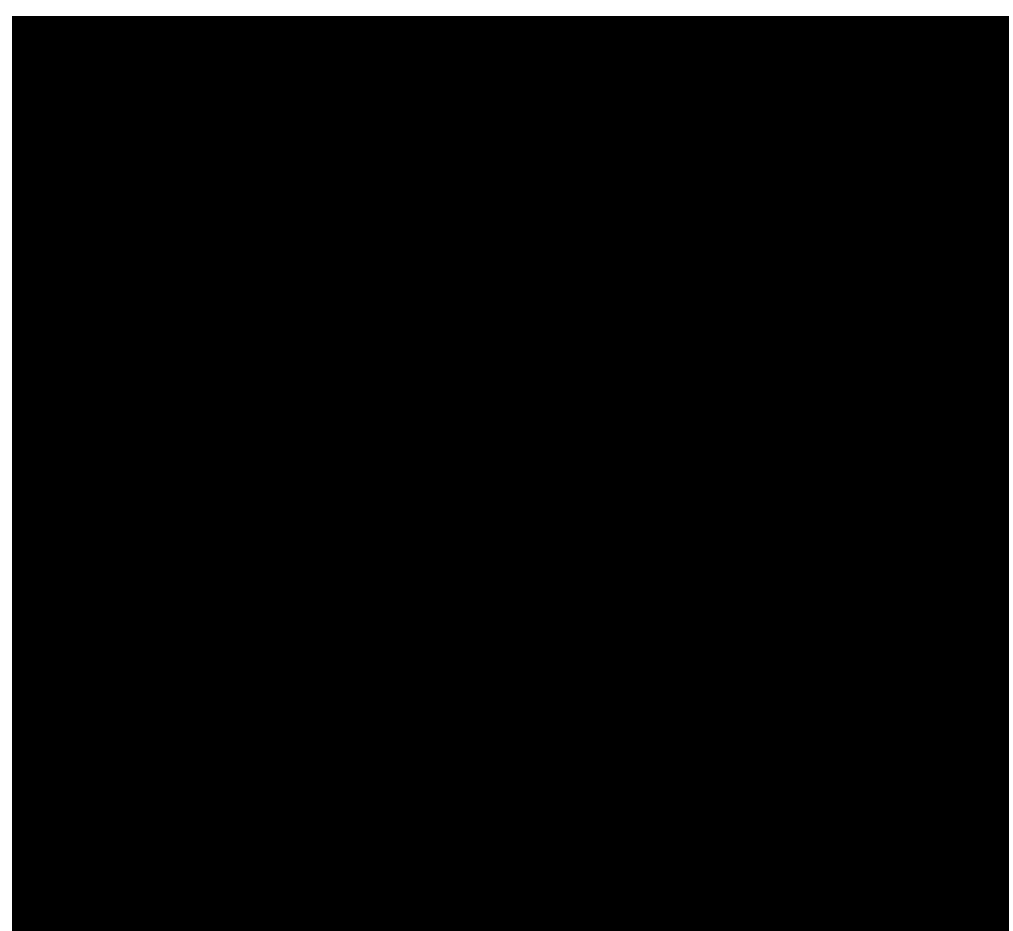

Fig. 6.5. Reflector requirements for a displaced polar orbit. Contours indicate radiation pressure acceleration $a\left(\mathrm{~mm} \mathrm{~s}^{-2}\right)$ and the dashed line indicates the stability boundary (stable orbits if $\rho>4 z$ ). 


\section{Displaced Polar Orbit Stability}

Now that a family of orbits with the appropriate reflector orientation has been defined, its stability characteristics will be investigated. This is achieved by linearising the equations of motion about the nominal orbit to obtain a variational equation. The variational equation then describes the motion of the reflector in the neighbourhood of the nominal orbit. The stability of the resulting trajectory may be determined by examining the eigenvalues of the variational equation. If any of the eigenvalues are real and positive, the motion of the reflector is exponentially divergent so that the orbit will be unstable. However, if all the eigenvalues are purely imaginary, the reflector is expected to remain bound in a neighbourhood of the nominal orbit. It should be noted that a linear analysis is performed, which in the present case provides only necessary conditions for stability, but sufficient conditions for instability.

The non-linear equations of motion will be linearised by perturbing the reflector from its nominal orbit with the reflector attitude fixed in the rotating frame of reference (McInnes, 1999). In addition, since the radiation field is uniform over the length-scale of the problem, the solar radiation pressure acceleration experienced by the reflector remains constant during the perturbation. Firstly, a perturbation $\delta=(\xi, \psi, \eta)$ will be added to the reflector position vector at some point $\mathbf{r}_{0}=\left(\rho_{0}, \theta_{0}, z_{0}\right)$ on the nominal orbit such that $\mathbf{r}_{o} \rightarrow \mathbf{r}_{o}+\boldsymbol{\delta}$. The variational equation is then obtained from the non-linear equations of motion as 


$$
\begin{gathered}
\frac{d^{2} \xi}{d t^{2}}-2 \omega \rho_{o} \frac{d \psi}{d t}+M_{11} \xi+M_{13} \eta=0 \\
\frac{d^{2} \psi}{d t^{2}}+\frac{2 \omega}{\rho_{o}} \frac{d \xi}{d t}=0 \\
\frac{d^{2} \eta}{d t^{2}}+M_{31} \xi+M_{33} \eta=0
\end{gathered}
$$

where the linear expansion coefficients are found to be

$$
\begin{gathered}
M_{11}=3 \omega^{2}+\widetilde{\omega}^{2}\left[1-3\left(\frac{\rho}{r}\right)^{2}\right] \\
M_{13}=M_{31}=-3 \tilde{\omega}^{2}\left[\frac{\rho z}{r^{2}}\right] \\
M_{33}=\tilde{\omega}^{2}\left[1-3\left(\frac{z}{r}\right)^{2}\right]
\end{gathered}
$$

Owing to the azimuthal symmetry of the problem all derivatives with respect to $\theta$ in the linear expansion will vanish. Therefore, the six terms $M_{2, \mathrm{j}}$ and $M_{\mathrm{j}, 2}(\mathrm{j}=1-3)$ are zero. The set of three coupled, linear ordinary differential equations defined by Eq. (6.13) may be reduced to two by integrating Eq. (6.13b) to obtain

$$
\frac{d \psi}{d t}=-\frac{2 \omega}{\rho_{o}}\left(\xi-\xi_{o}\right)
$$

This equation is in effect a linearised form of Kepler's third law, describing the orbital angular velocity of the reflector relative to the nominal orbit due to the radial displacement $\xi$. This equation may 
then be substituted into Eq. (6.13a) to eliminate the azimuthal term. However, this substitution then leads to a constant term $4 \omega^{2} \xi_{0}$ in Eq. (6.13a) so that the variational equation is no longer homogeneous. It can be shown that the non-homogeneity can be easily removed by re-scaling the co-ordinates through a change of variable

$$
\begin{gathered}
\xi^{\prime}=\xi-\frac{4 \omega^{2} M_{33}}{M_{11} M_{33}-M_{13} M_{31}} \xi \\
\eta^{\prime}=\eta+\frac{4 \omega^{2} M_{13}}{M_{11} M_{33}-M_{13} M_{31}} \xi
\end{gathered}
$$

Using this transformation a reduced variational system with a set of two coupled equations is then obtained as

$$
\frac{d^{2}}{d t^{2}}\left[\begin{array}{l}
\xi^{\prime} \\
\eta^{\prime}
\end{array}\right]+\left[\begin{array}{ll}
M_{11} & M_{13} \\
M_{31} & M_{33}
\end{array}\right]\left[\begin{array}{l}
\xi^{\prime} \\
\eta^{\prime}
\end{array}\right]=\left[\begin{array}{l}
0 \\
0
\end{array}\right]
$$

with the reflector free to drift along the nominal orbit in the azimuthal direction.

The stability of the family of orbits defined in Section 4 may now be investigated by calculating the eigenvalues of the variational equation. The eigenvalues may be obtained in the usual manner by substituting an exponential solution of the form

$$
\left[\begin{array}{l}
\xi^{\prime} \\
\eta^{\prime}
\end{array}\right]=\left[\begin{array}{l}
\xi_{o} \\
\eta_{o}
\end{array}\right] \exp (\lambda t)
$$

Substituting this trial solution into Eq. (6.17) yields a matrix equation of the form 


$$
\left[\begin{array}{cc}
\lambda^{2}+M_{11} & M_{13} \\
M_{31} & \lambda^{2}+M_{33}
\end{array}\right]\left[\begin{array}{l}
\xi_{o} \\
\eta_{o}
\end{array}\right]=\left[\begin{array}{l}
0 \\
0
\end{array}\right]
$$

For non-trivial solutions it is required that the determinant of this matrix equation vanishes. The characteristic polynomial of the variational equation is then found to be

$$
\lambda^{4}+\operatorname{tr}(\mathbf{M}) \lambda^{2}+\operatorname{det}(\mathbf{M})=0
$$

The stability characteristics may now be investigated by substituting for the appropriate functional form of $\omega$ from Eq. (6.12). Then, regions in the $\rho$-z plane may be identified where the roots of the characteristic polynomial are purely imaginary with $\lambda_{j}{ }^{2}<0 \quad(j=1-4)$ indicating stable, bound orbits. Such purely imaginary eigenvalues are obtained if $\operatorname{tr}(\mathbf{M})>0$ and $\operatorname{det}(\mathbf{M})>0$, again noting that only a linear analysis is presented which provides necessary condition for stability, but sufficient conditions for instability.

The family of displaced orbits is partitioned into stable and unstable groups by the conditions $\operatorname{tr}(\mathbf{M})>0$ and $\operatorname{det}(\mathbf{M})>0$, denoted by the dashed line in Fig. 6.5. This partition between the stable and unstable orbits is approximated by the boundary $\rho=4 z$, with stable orbits in the region $\rho>4 z$. It can be seen then that only orbits relatively close to the planetary terminator are linearly stable, while orbits with a large displacement are unstable. Clearly, it is advantageous to use stable orbits to avoid the need for active control and to mitigate against the risk of failure. Any failure in such an active control system would excite the unstable modes of the orbit and lead to 
a catastrophic loss of the reflector system. However although unstable, it can be shown that the orbits are strictly controllable (McInnes, 1999).

\section{Solar Reflector Fabrication}

The mass required to fabricate a solar reflector utilizing the passively stable orbit discussed above will now be considered. For an orbit with a radius $\rho$ of 15 Mars radii and a displacement $z$ of 2 Mars radii in the anti-Sun direction, Eq. (6.10b) shows that an acceleration of $5.05 \mathrm{mms}^{-2}$ is required. If an orbit with a smaller displacement is considered then the required reflector mass will rise sharply, while a larger displacement will soon lead to an unstable orbit as the partition between the stable and unstable orbits is reached. At the mean distance of Mars from the Sun the solar radiation pressure $P$ exerted on a perfectly absorbing surface is $1.964 \times 10^{-6} \mathrm{Nm}^{-2}$. For an ideal specular reflector of mass per unit area $\sigma$, the resulting acceleration $a$ is just $2 P / \sigma$. Therefore, to generate an acceleration of $5.05 \mathrm{mms}^{-}$

${ }^{2}$, the required mass per unit area $\sigma$ of the reflector will be $1.01 \mathrm{gm}^{-2}$. This is a value similar to the canonical $1 \mathrm{gm}^{-2}$ requirement for high performance deployable solar sails (McInnes, 1999). The pitch of the solar reflector $\alpha$ can also be obtained from Eq. (6.11) and is found to be $41.2^{\circ}$. The required reflector area to obtain a given increase in solar insolation $\dot{Q}$ can therefore be obtained from $\dot{Q} / S \cos \alpha$. For a $30 \%$ increase in total insolation $\left(6.38 \times 10^{15} \mathrm{~W}\right)$ the 
reflector area is found to be $1.44 \times 10^{13} \mathrm{~m}^{2}$. Again, a large number of reflectors are envisaged, distributed azimuthally about the displaced orbit to provide the total required surface area. The mass of the reflector can now be obtained from the required reflector mass per unit area as $\sigma \dot{Q} / S \cos \alpha$, where $\sigma$ is obtained from the required reflector acceleration as $2 P / a$. For the orbit of interest the total reflector mass is found to be $1.120 \times 10^{10} \mathrm{~kg}$. A comparison with large-scale terrestrial engineering ventures in shown in Table 6.1, while a comparison with space-based geo-engineering schemes is shown in Table 6.2 .

Clearly, the fabrication of a reflector with a total mass of order $10^{10} \mathrm{~kg}$ would require an advanced industrial space capacity with the ability to exploit extraterrestrial resources. For example, the mass requirements for the solar reflector can be satisfied by M-type asteroids, which are relatively abundant in Nickel-Iron materials (Gehrels, 1979). Assuming that the reflector is fabricated from thin metallic film processed from such a body, and that the asteroid has a bulk density similar to that of Iron $\left(7860 \mathrm{~kg} \mathrm{~m}^{-3}\right)$, an asteroid with a radius of order $70 \mathrm{~m}$ will provide the required mass. Including a non-metallic content of as much as $50 \%$ will increase the required asteroid radius to order $90 \mathrm{~m}$. The asteroid would require to be processed in-situ, probably using solar heating, and the metallic products extruded into thin film for fabrication of the solar reflector elements. Again, assuming a density of order $1 \mathrm{gm}^{-2}$, the film thickness would be of order $0.1 \mu \mathrm{m}$. Processing thin film of this thickness is clearly 
challenging, but ultimately it is the film thickness which drives the total mass of the reflector system. Terrestrial experiments have been performed to produce thin metallic film of comparable thickness, although only on a laboratory scale (Drexler 1979).

Lastly, it is interesting to speculate as to the effect of such a displaced ring of orbiting solar reflectors on the sky, as seen from the surface of Mars. For an observer near the equator, the tip of the ring will slowly rise above the eastern horizon, and would likely be rather dim in the darkening dusk sky due to diffusely scattered light. Since the ring is composed of a vast number of individual reflectors, it would like appear as a dense ribbon of point sources, packed denser than the Pleiades. As local sunset is reached the ring would brighten relative to the darkening sky and gradually rise until it formed a thin bright arc stretching from the northern horizon to the south. Since the orbit of the ring is displaced, the peak brightness of the ring would occur sometime after sunset, providing a spectacularly bright structure in the night sky. After peak brightness, the ring would begin to dim, drifting westwards and sinking again towards the horizon. This spectacular display would then be repeated prior to dawn with the ring providing a brilliant precursor to local sunrise.

Table 6.1. Mass comparison with terrestrial engineering ventures

\begin{tabular}{lll}
\hline Scale & Mass $(\mathrm{kg})$ & Engineering venture \\
\hline $10^{8}$ & $6.5 \times 10^{8}$ & 'Knock Nevis’ oil tanker (fully laden) \\
$10^{8}$ & $6 \times 10^{9}$ & Great pyramid of Giza \\
$10^{10}$ & $6 \times 10^{10}$ & Concrete used for Three Gorges dam \\
$10^{11}$ & $2 \times 10^{11}$ & Water stored in London’s reservoirs \\
$10^{12}$ & $7 \times 10^{12}$ & World annual $\mathrm{CO}_{2}$ emissions \\
\hline
\end{tabular}


Table 6.2. Mass comparison with terrestrial geo-engineering

\begin{tabular}{llll}
\hline Geoengineering concept & Mass $(\mathrm{kg})$ & Area $\left(\mathrm{m}^{2}\right)$ & Density $\left(\mathrm{g} \mathrm{m}^{-2}\right)$ \\
\hline Struck (Lunar $\mathrm{L}_{4 / 5}$ dust cloud) & $2.1 \times 10^{14}$ & - & - \\
Pearson (Earth orbit dust ring) & $2.3 \times 10^{12}$ & $1.10 \times 10^{14}$ & - \\
McInnes A (Solar L $\mathrm{L}_{1}$ reflecting disks) & $2.6 \times 10^{11}$ & $6.57 \times 10^{12}$ & 40.2 \\
McInnes B (Solar L $\mathrm{L}_{1}$ absorbing disks) & $5.2 \times 10^{10}$ & $6.57 \times 10^{12}$ & 7.9 \\
Angel (Solar $\mathrm{L}_{1}$ refracting disks) & $2.0 \times 10^{10}$ & $4.70 \times 10^{12}$ & 4.2 \\
\hline
\end{tabular}

\section{Conclusions}

Large-scale Mars terraforming using orbiting solar reflectors has been considered. In particular, a family of displaced planetary orbits has been presented which are suitable for use by solar reflectors as part of a large Mars terraforming effort. The orbits are configured to ensure that solar radiation is continually reflected onto the planetary surface to increase the total solar insolation. A particularly useful stable family of orbits has been identified which lie close to the planetary terminator. For comparison, static equilibria have been considered as potential locations for Mars solar reflectors. However, the reflector orientation required to ensure static equilibrium leads to significant losses of reflected solar radiation which is not intercepted by the planetary body. Static equilibria therefore have serious problems for use by Mars solar reflectors, while displaced orbits can be configured to be Sun-synchronous and passively stable leading to benefits for their use in large-scale terraforming.

\section{References}

Angel R (2006) Feasibility of cooling the Earth with a cloud of small spacecraft near the inner Lagrange point $\left(\mathrm{L}_{1}\right)$. Proc. Nat. Acad. Sci. 103, 17184-17189. 
Badescu V (2005) Regional and seasonal limitations for Mars intrinsic ecopoiesis. Acta Astronautica. 56, 670-680.

Birch P (1992) Terraforming Mars quickly. J British Interplanetary Society. 45, 331-340.

Bookless J and McInnes CR (2004) Dynamics, stability and control of displaced non-Keplerian orbits, IAC-04-A.7.09, 55th International Astronautical Congress, Vancouver.

Drexler KE (1979) High Performance Solar Sails and Related Reflecting Devices, $4^{\text {th }}$ Princeton/AIAA Conference on Space Manufacturing Facilities, Princeton, Paper AIAA-79-1418.

Early JT (1989) Space-based solar shield to offset greenhouse effect, J British Interplanetary Society. 42, 567-569.

Fogg M (1992) A synergestic approach to terraforming Mars. J. British Interplanetary Society. 45, 315- 329.

Fogg MJ (1995) Terraforming: Engineering Planetary Environments, SAE International, ISBN 1-56091-609-5.

Forward RL (1991) Statite: A Spacecraft That Does Not Orbit. J Spacecraft and Rockets. 28, 606-611.

Gehrels T (1979) Asteroids. Univ. of Arizona Press, Tucson.

Gerstell MF, Francisco JS, Yung YL, Boxe C and Aaltonee ET (2001) Keeping Mars warm with new super greenhouse gases, Proc. Natl. Acad. Sci. USA. 98, 2154-2157.

Mautner M and Parks K (1990) Space-based control of the climate, Proc. Space 90, Amer. Soc. Civ. Eng. 1159-1169.

McInnes CR (1991) Solar Sail Halo Trajectories: Dynamics and Applications, $42^{\text {nd }}$ International Astronautical Congress, Montreal, Paper IAF-91-334.

McInnes CR and Simmons JFL (1992) Halo Orbits for Solar Sails II - Geocentric Case. J Spacecraft and Rockets, 29, No. 4, 472-479.

McInnes CR, McDonald AJC, Simmons JFL and MacDonald EW (1994) Solar sail parking in restricted three-body systems. J Guidance Dynamics and Control, 17, 399-406. 
McInnes C.R (1999) Solar Sailing: Technology, Dynamics and Mission Applications, Springer-Verlag Series in Space Science and Technology, SpringerVerlag, ISBN 1-85233-102-X.

McInnes CR (2002) Non-Keplerian orbits for Mars solar reflectors. J British Interplanetary Society. 55, 74-84.

McInnes CR (2006) Planetary Macro-engineering using Orbiting Solar Reflectors', in Macro-Engineering: A Challenge for the Future (ed. V. Badescu, R. Cathcart and B. D. Schuiling), Springer-Verlag, ISBN 1-402-03739-2, pp. 215250.

McKay CP, Toon, OB and Kastling JF (1991) Making Mars habitable. Nature, 352, 489-496.

Oberg JE (1981) New Earths: Restructuring Earth and Other Planets, New American Library Inc, New York.

Sagan C (1961) The planet Venus. Science, 133, 849-858.

Sagan C (1973) Planetary engineering on Mars. Icarus. 20, 513-514.

Zubrin R and McKay C (1997) Technological requirements for terraforming Mars, J British Interplanetary Society. Soc. 50, 83-92. 